\title{
Creating a Culture of Equity: Building Awareness Within the Montgomery County Department of Health and Human Services
}

Surbhi Sardana, Montgomery County Department of Health and Human Services, Maryland, USA

\begin{abstract}
The Robert Wood Johnson Foundation recognizes Montgomery County as the county with the best health outcomes, length of life, and quality of life in all of Maryland. While impressive, the overall statistics do not reflect the disparities among certain population groups in the county. As a major provider of safety net health and social services to county residents, the Montgomery County Department of Health and Human Services (MCDHHS) wants to empower staff to think and act differently to generate better outcomes for disadvantaged communities. Among the many phases toward building an equity value driven organization, the Department felt that cultivating a common understanding and buy-in from all levels of staff is critical to a cultural shift. In 2014, a comprehensive workshop was implemented to raise awareness and encourage courageous conversations. The workshop seeks to create a common understanding of equity, its principles and applied strategies, and provide Department staff with the tools to treat their colleagues, customers, and clients more equitably. To date, 828 staff, contractors, and community partners have completed the workshop and 47 staff members have trained as workshop peer facilitators. This paper will explore in more detail the information-related factors and processes present in the workshop and their equity impact on the Department's practices.
\end{abstract}

Keywords: employee training; equity; health justice; local health department

Publication Type: special section publication

\section{Introduction}

M ontgomery County, Maryland is a large urban/suburban county with a population of one million plus residents. As a majority-minority county, it is unique in its cultural and ethnic diversity. Census data shows that over $55 \%$ of residents identify with a race/ethnicity other than non-Hispanic white. Almost one-third of county residents are foreign-born, hailing mostly from Asia, Africa, and Latin America. Based on these statistics, it is not surprising that almost $40 \%$ of county residents speak a language other than English at home. (Robert Wood Johnson Foundation, 2017).

Montgomery County is also perceived as a well-educated, affluent county producing top health outcome ratings. The Robert Wood Johnson Foundation (2017) ranks the county number one in the state for health outcomes such as quality of life, health behaviors, clinical care, social and economic factors, and physical environmental factors. While impressive, the overall statistics do not reflect the disparities among certain population groups within the county. Subpopulations reveal significant differences in health outcomes as well as barriers to access to care. County

The International Journal of Information, Diversity, \& Inclusion, 3(3), 2019

ISSN 2574-3430, jps. library.utoronto.ca/index.php/ijidi/index

DOI: $10.33137 /$ ijidi.v3i3.32966 
ratings and census data can mask the patterns of poverty, lack of self-sufficiency, uninsured rates, housing burden and other social determinants affecting disadvantaged communities in the county.

Indeed, there are many underlying causes to the disparate statistics. As a major provider of safety net health and social services to county residents, the Montgomery County Department of Health and Human Services (MCDHHS) aims to empower staff to generate better, more equitable outcomes for disadvantaged communities by challenging assumptions and using a more holistic lens to understand social, political, and environmental impacts on communities. In 2009, the Department began to look at adopting an equity and social justice frame to guide its transformation. Among the many phases toward building an equity value driven organization, the Department felt that cultivating a common understanding and buy-in from all levels of staff was critical to a cultural shift leading to the development of the "Creating a Culture of Equity" workshop.

To explore the challenges and rewards of offering this workshop, this article will begin with a literature review to outline the magnitude of this issue across the U.S. It will then discuss some of the details of planning and executing this workshop, so other organizations can use this information and incorporate it into their employee training program. Finally, it will present future directions and ideas regarding the expansion of this program.

\section{Literature Review}

Health disparities, determinants of wellbeing, and inequities have long been researched and reported at length by various mediums. Disparities based on race, socioeconomic status (SES), geography, age, sexual orientation, disability status, language, citizenship status, and many other characteristics persist in the U.S. (Orgera \& Artiga, 2018). These disparities become inequitable if they are avoidable, unfair, and unjust (Whitehead, 1992), and lead to "systematic differences in the health status of different population groups" (World Health Organization, 2017).

In the U.S., there are significant disparities steeped in racial and ethnic differences. The Centers for Disease Control and Prevention (CDC) reports the infant mortality rate was 2.8 times higher among non-Hispanic black mothers than among non-Hispanic Asian or Pacific Islander mothers in 2015. In 2016, low infant birthweight was also more prevalent among non-Hispanic black mothers and Puerto Rican mothers than any other racial or ethnic group. Between 2006 and 2016, life expectancy for non-Hispanic blacks was seven years less than the highest life expectancy group among Hispanics (National Center for Health Statistics, 2018). The existence of severe racial and ethnic disparities in the U.S. is not disputed; however, these statistics should not undermine differences in health status based on other characteristics. In achieving health equity, the longterm vision of public health practitioners is to reduce or eliminate disparities that are avoidable and unfair.

Health disparities and inequities are inextricably linked with "social, economic, or environmental disadvantage," or in other words, the determinants of health (U.S. Department of Health and Human Services, Office of Disease Prevention and Health Promotion, n.d.). In fact, Healthy People 2020 added societal determinants of health to their health promotion and disease prevention objectives. Societal determinants of health consider the interaction between physical and social conditions, in addition to policies made across governmental and non-governmental

The International Journal of Information, Diversity, \& Inclusion, 3(3), 2019

ISSN 2574-3430, jps.library.utoronto.ca/index.php/ijidi/index

DOI: $10.33137 /$ ijidi.v3i3.32966 
sectors (Secretary's Advisory Committee on National Health Promotion and Disease Prevention Objectives for 2020, 2010).

Local health departments (LHDs) are in a strategic position to impact the determinants of health and wellbeing, as they are connected to the community they serve and understand the local "assets, needs and culture" (Sokol et. al, 2017, p. 118). The Big Cities Health Coalition is a "forum for the leaders of America's largest metropolitan health departments to exchange strategies and jointly address issues to promote and protect the health and safety" of the public (Big Cities Health Coalition, n.d.). For members of the Big Cities Health Coalition, health equity is a high priority and the determinants of health are an "optimal approach" to health improvements (Narain et al., 2018). However, perceived barriers to health equity within LHDs include "limited training and guidance on how to conduct this type of work" (Narain et al., 2018, p. 342). The commitment and skills needed to promote health equity can trickle down the organization, from the top down. Strong leaders who are "effective at managing change" can encourage work units to actively work towards health equity (Furtado et al., 2018, p. 44).

Nonetheless, the siloed nature of equity work can be limiting to organizational change. Often, health equity is viewed as the work of minority health, health equity, and health disparities centers within a department. Isolating health equity work can perpetuate the perception that non-community health staff, such as administrative, information technology, contracts, and fiscal staff, do not play a role in improving the health of the community (Furtado et al., 2018). However, as the American Public Health Association (APHA) states, health equity is "a framework within which public health practitioners from all disciplines can work" (2015). In order to create this framework, LHDs must build "knowledge, understanding and capacity" to achieve health equity (APHA, 2015).

Health equity toolkits for LHD implementation are available. The Bay Area Regional Health Inequities Initiative (BARHII) created a toolkit for its coalition of San Francisco Bay Area public health departments. The Organization Self-Assessment for Addressing Health Inequities Toolkit provides LHDs with a guide to "identify the skills, organizational practices and infrastructure needed to address health equity" (BARHII, 2010). The National Association of County \& City Health Officials (NACCHO) published their Health Equity and Social Justice program to "advance the capacity of LHDs to confront the root causes of inequities in the distribution of disease and illness" (2016). The program includes a web-based course, toolkit, and a handbook.

This paper explores an equity training conducted by the Montgomery County Department of Health and Human Services in Maryland in order to create knowledge and understanding of health equity concepts among Department staff.

\section{“Creating a Culture of Equity" Workshop}

In 2014, a comprehensive workshop was implemented to raise awareness and encourage courageous conversations. The workshop seeks to create a common understanding of equity, its principles and applied strategies, and provide Department staff with an equity lens to use in their daily work. This paper will explore in more detail the information-related factors and processes presented in the workshop and the resulting equity impact on the Department's practices.

The International Journal of Information, Diversity, \& Inclusion, 3(3), 2019

ISSN 2574-3430, jps.library.utoronto.ca/index.php/ijidi/index

DOI: $10.33137 /$ ijidi.v3i3.32966 


\section{Developing the Workshop Curriculum}

MCDHHS contacted CommonHealth ACTION to begin equity work in the Department. CommonHealth ACTION is a non-profit based in Washington D.C. that "tailor[s] learning experiences, group processes, and organizational planning" to help organizations "map, manage, measure, and master their change" (CommonHealth ACTION. n.d.) in pursuit of health equity. As a result of this relationship, the Department completed a logic model in 2012 that outlined an intent to provide training to management and staff on the equity principles and social determinants of health. In 2013, CommonHealth Action provided the Department with a training curriculum. The logic model and the training both arose from the Theory of Change.

The Theory of Change is an approach by which a program or intervention achieves long-term goals "through a logical sequence of intermediate outcomes" (Lund, Lee, Breuer, \& De Silva, 2016, p. 2). The Theory uses a "backwards mapping approach" in which long-term goals are first identified, then the series of actions, activities, and short- and mid-term outcomes needed to achieve the long-term goals are determined.

For the "Creating a Culture of Equity" workshop, the identified goals are listed below:

1. Create awareness and expand knowledge about equity amongst all levels of staff within the department.

2. Provide staff with the language to communicate with colleagues, partners, and clients about equity.

3. Create a shared understanding of equity amongst MCDHHS leadership and staff and apply strategies with regards to policies, practices, procedures, and infrastructure.

4. Build a cohort of staff that will be able to expand the capacity of equity champions in the department.

5. Encourage a culture to support innovation, collaboration, and continuous learning throughout the department.

The objectives for this workshop include:

1. Establish a shared language to support communication, knowledge sharing, and collaboration amongst colleagues, partners, and clients.

2. Recognize the impacts of the determinants of well-being and equity on the individuals and community you serve in Montgomery County.

3. Apply strategies for putting equity into practice.

4. Transfer this equity knowledge practice and experience to colleagues and partners.

The first draft of this training curriculum was presented to the Department's Senior Leadership Team (SLT) as a pilot. After extensive feedback from the SLT, the Department took on the

The International Journal of Information, Diversity, \& Inclusion, 3(3), 2019

ISSN 2574-3430, jps.library.utoronto.ca/index.php/ijidi/index

DOI: $10.33137 /$ ijidi.v3i3.32966 
responsibility of revising and updating the curriculum to better suit the staff's needs. After much revision, the training was presented to Department staff and management in 2014.

\section{Implementing the Workshop Curriculum}

\section{Recruitment}

Before implementation of the workshop could begin, facilitators were recruited and trained on the curriculum material in February and March of 2014. All workshop peer facilitators are Department staff who volunteer their time to the equity cause. Recruitment of peer facilitators creates a better vehicle for sustainable learning. The facilitators undergo extensive coaching before each workshop and dedicate hours of research to equity and other related concepts.

\section{Advertisement}

The Center for Continuous Learning (CCL) Program Catalog advertises trainings for Department staff, contractors, and community partners. The CCL program offers "board-approved continuing education classes for social workers and therapists, as well as non-credit courses" (Montgomery County Government, Office of Human Resources, n.d.). Email distribution lists and word of mouth are additional mediums for advertisement of the workshop.

\section{The Curriculum}

The "Creating a Culture of Equity" workshop is a one-day training available to Department staff and Montgomery County partners led by the Department staff volunteers. The workshop facilitators use different methods for adult learning, such as training and facilitation methods along with audio and visual learning. Handouts, video clips, PowerPoint slides, group discussions and group activities are all integrated to create a well-rounded experience.

The workshop is divided into four modules: Modules 1 and 2 set the stage for Modules 3 and 4 . The first two modules are deemed "training" or "teaching" modules. Peer facilitators create a common language and understanding of equity that is continuously reinforced throughout the day. Modules 3 and 4 encourage staff to consider how their clients and customers in Montgomery County experience inequities. Workshop staff work collaboratively to discuss strategies within their "sphere of influence" that can positively impact outcomes for a client. Many areas within the county are impacted by poverty, housing burdens, and uninsurance rates. County staff encounter many of these clients as social work cases, therapy patients, and as applicants for government benefits.

Module 1. Peer facilitators lay out the goals and objectives and seek agreement on a basic set of ground rules for all participants and facilitators to follow. As stated earlier, Module 1 begins to cultivate a common language and understanding of equity concepts including equity lens, equity vs. equality, perspective transformation, community, and social determinants of health and wellbeing.

Module 1 utilizes different teaching methods to explain conceptual terms. For example, peer facilitators illustrate the difference between equality and equity in two major ways: the first method invites participants to partake in a storytelling experience by which participants are treated "equally" and discuss what a more equitable situation could look like. In the second

The International Journal of Information, Diversity, \& Inclusion, 3(3), 2019

ISSN 2574-3430, jps.library.utoronto.ca/index.php/ijidi/index

DOI: $10.33137 /$ ijidi.v3i3.32966 
method, facilitators explain a popular image of three boys of varying heights attempting to watch a baseball game over a wooden fence. In the first image, each boy is given a box on which he can stand to see the game (equality), yet the shortest boy is still unable to view the game. In the second image, the tallest boy is not given a box - he can see clearly over the fence without the support. The medium-height boy only needs one box support to see over the fence. The shortest boy receives the greatest number of supports to see the game. This is explained as equity - everyone's needs are considered in the distribution of resources. In the last image, the wooden fence is replaced by a steel fence that allows all boys to clearly view the game without supports. Facilitators explain this image as the removal of structural or systemic barriers that can be accomplished through buy-in at all levels, and through implementation of equitable policies.

Module 2. Module 2 continues to build upon the equity concepts from Module 1 . Module 2 looks at disparities vs. inequities, defines disproportionalities and illustrates the impact of social determinants of health within the county. Module 2 also incorporates a "Place Matters" video clip from the 2008 docuseries Unnatural Causes (California Newsreel \& Vital Pictures, Inc., 2008). The video is effective at explaining the health impact of neighborhood and community factors in low-income communities like Richmond, California. Facilitators use the video as a segue into the social determinants of health affecting county residents. In other words, through this video, facilitators try to explain how "place matters" plays out in Montgomery County (U.S. Census Bureau. n.d.).

Other materials used in Module 2 are statistics from the American Community Survey that illustrate the patterns of poverty, lack of education, impact of long commutes, housing burden, and uninsured rates across Montgomery County. Statistics are transformed from data into a visual story via color-coded maps that are effective at depicting the patterns of social determinants across the County. These patterns explain the daily challenges vulnerable county populations face, many of whom Department staff see in their office for various social services.

Module 3. Module 3 begins with a brief review of milestones in the history of civil rights in the U.S. The history gives context to the concept of the existing "-isms" in society, such as racism or sexism, and to the idea of privilege and oppression in the contemporary society. In this portion, two video clips on race and racism in the U.S. often spur discussions of personal and professional experiences (Foster \& Stephenson, 2015). Workshop participants often use these experiences to raise awareness of the societal realities of disadvantaged populations.

The idea of privilege and oppression leads participants into an interactive activity titled "The Walk." In a visually palpable way, "The Walk" allows participants to experience the advantages and disadvantages society affords to different groups of people. Participants are asked to selfidentify based on a list of characteristics. For each category, participants are asked to take a step forward or backward to signify an advantage or disadvantage in society, depending on the specific characteristic they self-identify with.

"The Walk" allows participants to reflect not only on their own access to personal privilege or experience of oppression, but also the situations facing their clients and customers. Participants walk in the shoes of entire communities in Montgomery County that share similar characteristics, experiences, and barriers.

Module 4. Module 4 is the final and culminating module of the workshop. The intent is to provide

The International Journal of Information, Diversity, \& Inclusion, 3(3), 2019

ISSN 2574-3430, jps.library.utoronto.ca/index.php/ijidi/index

DOI: $10.33137 /$ ijidi.v3i3.32966 
Department staff with the tools and strategies to apply equity to their work. Module 4 occurs in three group activities mirroring the sort of teamwork staff may encounter in their workplace.

The first activity asks staff to identify the areas they feel are of the highest concern or priority in their community or clients' perspectives, in their local county-wide perspective, and in their personal perspective. This exercise affirms the importance of aligning these perspectives for the benefit of the community. In order to alleviate or solve the concerns facing the county, identifying the underlying social determinants is an important step.

The next activity takes a deeper dive into the challenges facing the community. Real-time scenarios that have been observed in the county are provided to staff. Utilization of data and scenarios from staff's own work environment encourages staff buy-in at all levels. Staff are then tasked with the challenge of getting to the root cause of these scenarios using a technique often used in the business community titled "But Why" (Community Tool Box, n.d.). The goal of this technique is to understand the inequity further upstream, leading to the identification of relevant social determinant(s) affecting the community or individual. This technique encourages staff to think about all the possible concerns and stressors facing their client(s), in addition to the prevalent issue they are addressing.

The last exercise asks staff to brainstorm a few strategies within their sphere of influence that could positively impact the scenarios from the previous activity. The goal of this exercise is to encourage staff to start thinking about small changes in their own work that could positively impact their client's or customer's challenges. Although not all staff have the ability to change or implement policies, there are small steps that can effect change at all levels. Participants have suggested increased collaboration and integration across service areas, access to reduced fees for services, and bus tokens to reduce the burden of transportation costs as just a few of the strategies that can be acted upon.

\section{Impact and Next Steps}

\section{Evaluations}

$\mathrm{CCL}$ issues a standard evaluation for all the classes and trainings under its purview. Since the implementation of the "Creating a Culture of Equity" workshop, evaluations have been collected from program participants. CCL requires completed evaluations to be returned to them in a report after each workshop. The evaluations are not mandatory for participants, although they are highly encouraged. The evaluations include three main sections: (a) rating scale from 1 to 5 to evaluate each facilitator; (b) rating scale from 1 to 5 to evaluate participant experience; and (c) open-ended questions to solicit comments. The evaluations are compiled in a report at the end of the calendar year and the results are communicated to all facilitators and team staff. End-of-year reports were compiled for each year the workshop was implemented with the exception of 2017, due to staff turnover.

Much of the feedback from the evaluations as well as the continued level of participation in these courses (which often leads to registration waiting lists) indicate that Department staff and partners want to hear and learn about equity concerns in the community. A sample of frequent responses to the open-ended questions are provided below.

1. What did you find most useful in today's session?

The International Journal of Information, Diversity, \& Inclusion, 3(3), 2019

ISSN 2574-3430, jps.library.utoronto.ca/index.php/ijidi/index

DOI: $10.33137 /$ ijidi.v3i3.32966 
- "The privilege walk was an eye opener."

- “The 'But Why' technique was useful.”

- "The videos provided a helpful visual learning tool."

2. What aspects of the content/presentation would you suggest changing or improving?

- "Nothing."

- "I would suggest more time for discussion with and among the audience."

3. What information, strategies, or techniques will you apply?

- "Asking 'But Why?' when clients come in order to get to the root causes."

- "All of it."

- "Encourage staff to use their equity lens."

4. What other learning on this topic or other topics would enhance your job performance?

- "More application methods."

\section{Challenges}

Over the course of the development and implementation phases of this workshop, three major challenges were identified. The Department has worked scrupulously to transform the training to be more inclusive of all health and social justice issues. The original versions of the curriculum revolved around the concept of racial equity; however, the Department has acknowledged that many other societal and systemic factors in addition to race affect the community. As such, collaboration and input from various individuals in the Department with various roles was collected to revise the training. To this day, the curriculum continues to evolve to address the concerns and priorities of the community. One of the strengths of this workshop is its highly adaptive nature. The content can be changed to reflect new and different equity concerns.

Another challenge is retention of peer facilitators. As is often heard about governmental organizations, time and resources are limited. Peer facilitation requires a dedication of time in addition to the facilitators' full-time positions. Retention of talented facilitators is an ongoing conversation because the training would not be the success it is today without the dedication of staff.

The third challenge is achieving buy-in at all levels of the Department. Although a significant proportion of staff, contractors, and partners have attended the workshop, there are still a significant number of individuals who have not. Even among some previous attendees, there remains some misinformation or doubt regarding the importance of equity. Other methods to achieve greater understanding of equity principles will need to be considered.

The International Journal of Information, Diversity, \& Inclusion, 3(3), 2019

ISSN 2574-3430, jps.library.utoronto.ca/index.php/ijidi/index

DOI: $10.33137 /$ ijidi.v3i3.32966 


\section{Limitations}

This training has been implemented for a local health department in a very unique county. The diversity in Montgomery County is not paralleled everywhere across the U.S. or the globe. As such, the content of the training may not be suitable for all settings in terms of applicable content, comprehension level, length, and language. However, the basic equity principles and idea of fairness for all is a tenet that can be accepted across cultures, geographies, and languages. A strength of this training is the flexible nature of the content. Facilitators or training coordinators in other settings can easily mold the basic information, information processes, and information mediums to reflect the societies they are addressing.

Another limitation that is important to address are the CCL evaluations. As mentioned earlier, the evaluations are not mandatory which can impact the response rate and the quality of responses, such as participant comments on the qualitative questions.

\section{Next Steps}

From 2014 through 2018, over one-third of the 1,700 MCDHHS staff members have completed the "Creating a Culture of Equity" workshop and 47 members have been trained as workshop peer facilitators. A total of 33 workshops have been conducted reaching 828 participants, including MCDHHS staff, contractors, and community partners. To accommodate staff that are unable to leave their desk to attend a whole day training, the workshop is now also available virtually as a pre-scheduled live webinar for which staff can register to attend through CCL. The County's partners are also increasingly interested in partaking in the workshop experience. The Department has been receiving regular external requests for various elements of this presentation. Portions of the "Creating a Culture of Equity" workshop have been incorporated into the Department's "New Employee Orientation" since mid-2016 to introduce employees to the concept and to reinforce the culture of equity in the Department.

The Department will continue to offer the workshop to staff and community partners for as long as the demand for the training remains. The Department is also looking to expand its breadth of equity-related trainings and tools. As such, equity toolkits that will help to operationalize equity are being developed. Further trainings on hot topic issues, such as implicit bias, are also in the planning process. Although comprehensive, the workshop is just one of a series of actions to integrate equity into the Department's work. Other methods to continue to build awareness and to develop practical tools to support staff in integrating equity in their daily work are being planned in the next phase of the equity journey. Dissemination of data, equity terms and principles, and the "But Why" technique are only the first steps to instituting long-lasting, equitable change.

\section{Acknowledgements}

I would like to extend my gratitude to Betty Lam, Chief of Office of Community Affairs and Elena Alvarado, Contractor for Office of Community Affairs for their writing assistance and feedback that greatly improved the manuscript.

The International Journal of Information, Diversity, \& Inclusion, 3(3), 2019

ISSN 2574-3430, jps.library.utoronto.ca/index.php/ijidi/index

DOI: $10.33137 /$ ijidi.v3i3.32966 


\section{References}

American Public Health Association (APHA). (2015, March). Better health through equity: Case studies in reframing public health work. Retrieved from https://www.apha.org/ /media/files/pdf/topics/equity/equity_stories.ashx

Bay Area Regional Health Inequities Initiative (BARHII). (2010). Organizational self-assessment toolkit. Retrieved from http://barhii.org/?s=toolkit\&submit=

Big Cities Health Coalition. (n.d.). Big Cities Health Coalition. Retrieved from http://www. bigcitieshealth.org/

California Newsreel (Producer). (2003). The house we live in: Race - the power of an illusion [Video file]. Retrieved from http://newsreel.org/video/RACE-The-House-We-Live-In

California Newsreel, \& Vital Pictures, Inc. (Producers). (2008). Unnatural causes. . . Is inequality making us sick? [Video file]. Retrieved from https: / / www.unnaturalcauses.org/

CommonHealth ACTION. (n.d.). Services. Retrieved from https://commonhealthaction.org/services/

Community Tool Box. (n.d.). Section 4. Analyzing root causes of problems: The "but why?" technique. Retrieved from https://ctb.ku.edu/en/table-of-contents/analyze/analyzecommunity-problems-and-solutions/root-causes/main

Furtado, K. S., Brownson, C., Fershteyn, Z., Macchi, M., Eyler, A., Valko, C., \& Brownson, R. C. (2018). Health departments with a commitment to health equity: A more skilled workforce and higher-quality collaborations. Health Affairs, 37(1), 38-46. https://doi.org/10.1377/hlthaff.2017.1173

Lund, C., Lee, L., Breuer, E., \& De Silva, M. (2016). Using theory of change to design and evaluate public health interventions: A systematic review. Implementation Science, 11(63), 1-17. https://doi.org/10.1186/s13012-016-0422-6

Montgomery County Government, Office of Human Resources. (n.d.). Programs and catalogs. Retrieved from https://www.montgomerycountymd.gov/HR/CareerDevelopment/TrainingPrograms.ht $\underline{\mathrm{ml}}$

Narain, K. D., Zimmerman, F. J., Richards, J., Fielding, J. E., Cole, B. L., Teutsch, S. M., \& Rhoads, N. (2018). Making strides toward health equity. Journal of Public Health Management and Practice, 25(4), 342-347. https://doi.org/10.1097/PHH.0000000000000852

National Association of County \& City Health Officials (NACCHO). (2016). Health equity and social justice. Retrieved from https://www.naccho.org/programs/public-healthinfrastructure/health-equity

National Center for Health Statistics. (2018). Health, United States, 2017. Retrieved from

The International Journal of Information, Diversity, \& Inclusion, 3(3), 2019

ISSN 2574-3430, jps.library.utoronto.ca/index.php/ijidi/index

DOI: $10.33137 /$ ijidi.v3i3.32966 
https://www.cdc.gov/nchs/data/hus/hus17.pdf

Orgera, K., \& Artiga, S. (2018, August 08). Disparities in health and health care: Five key questions and answers. Retrieved from https://www.kff.org/disparities-policy/issuebrief/disparities-in-health-and-health-care-five-key-questions-and-answers /

Robert Wood Johnson Foundation. (2017). County health rankings: Montgomery County, Maryland. Retrieved from

http://www.countyhealthrankings.org/app/maryland/2018/rankings/montgomery/cou nty/outcomes/overall/snapshot

Secretary's Advisory Committee on National Health Promotion and Disease Prevention Objectives for 2020. (2010, July 26). Healthy people 2020: An opportunity to address societal determinants of health in the United States. Retrieved from https://www. healthypeople.gov/2010/hp2020/advisory/SocietalDeterminantsHealth.ht $\underline{\mathrm{m}}$

Sokol, R., Moracco, B., Nelson, S., Rushing, J., Singletary, T., Stanley, K., \& Stein, A. (2017). How local health departments work towards health equity. Evaluation and Program Planning, 65, 117-123. https://doi.org/10.1016/j.evalprogplan.2017.08.002

Foster, B., \& Stephenson, M. (Directors). (2015). A conversation with white people on race [Video file]. The New York Times (Producer). Retrieved from https://www.nytimes.com/video/opinion/100000003773643/a-conversation-withwhite-people-on-race.html

U.S. Census Bureau. (n.d.). American FactFinder. Retrieved from http: //factfinder.census.gov

U.S. Department of Health and Human Services, Office of Disease Prevention and Health Promotion. (n.d.). Healthy people 2020. Retrieved from http://www. healthypeople.gov

Whitehead, M. (1992). The concepts and principles of equity and health. International Journal of Health Services, 22(3), 429-445. https:// doi.org/10.2190\%2F986L-LHQ6-2VTE-YRRN

World Health Organization. (2017, April 21). 10 facts on health inequities and their causes. Retrieved from https://www.who.int/features/factfiles/health_inequities/en/

Surbhi Sardana (surbhi.sardana@montgomerycountymd.gov) is a Community Health Program Coordinator for the Montgomery County Department of Health and Human Services in Maryland. As a Program Coordinator, Surbhi provides support for the equity training titled, "Creating a Culture of Equity" which encourages conversations about equitable practices in the workplace and with community members. She also supports the Equity Work Group and the Leadership Institute for Equity and Elimination of Disparities that work to expand the Equity and Social Justice framework the Department has adopted. Surbhi is also a candidate for a Master of Public Health from the University of Maryland, College Park.

The International Journal of Information, Diversity, \& Inclusion, 3(3), 2019

ISSN 2574-3430, jps.library.utoronto.ca/index.php/ijidi/index

DOI: $10.33137 /$ ijidi.v3i3.32966 